\title{
Sodium preferences and responses to sodium deficiency in rhesus monkeys
}

\author{
TERESA M. McMURRAY and CHARLES T. SNOWDON \\ University of Wisconsin, Madison, Wisconsin 53706
}

\begin{abstract}
Rhesus monkeys were offered sodium chloride $(\mathrm{NaCl})$ in various concentrations before and during the imposition of a dietary sodium deficiency. No evidence of a preference-aversion curve for $\mathrm{NaCl}$ appeared, providing support for the "dilute-water" hypothesis explaining sodium preference-aversion curves in rats. (Rhesus monkeys have separate sensory channels for water and salt, while rats have a combined water-salt sensory channel.) Although urinary and serum sodium levels were reduced during the period of sodium deficiency, there was no increase in the voluntary ingestion of $\mathrm{NaCl}$ during deficiency. However, three separate behavioral and physiological adaptations to sodium deficiency appeared among the monkeys.
\end{abstract}

One of the most common findings in studies of laboratory rats is that they show a preference for low concentrations of sodium chloride $(\mathrm{NaCl})$ relative to water up to isotonic concentrations, but reject higher concentrations (e.g., Bare, 1949). This aversion part of the curve is commonly explained by the postingestive osmotic effects of hypertonic saline (Mook, 1963). One hypothesis advanced to explain the preference part of the curve was the "dilute-water" hypothesis of Deutsch and Jones (1960). They noted that the rat does not have separate water- and saltsignaling fibers in the chorda tympani. The same cell signals both qualities (Zotterman, 1956). Given this anatomical feature, they argued that saline leads to less water signal per unit volume than does pure water. A greater volume of saline solutions is ingested because it takes a greater volume of saline solution to generate the same amount of "water" signal. Since the rhesus macaque has separate anatomical fibers to signal water and salts (Gordon, Kitchell, Ström, \& Zotterman, 1959), one would predict from the Deutsch and Jones hypothesis that rhesus macaques should not show any preferenceaversion functions for $\mathrm{NaCl}$, and that for thirsty monkeys distilled water should be the preferred solution.

A second common finding from studies of laboratory rats is that sodium-deficient rats show increased intake of more concentrated $\mathrm{NaCl}$ solutions than do nondeficient rats (Bare, 1949; Richter, 1939). A variety of techniques have been employed to make rats sodium deficient: subcutaneous injections of Formalin (Stricker, 1966; Jalowiec \& Stricker, 1970),

This study was supported in part by USPHS Grant AM 17. 171 to C.T.S. We thank Dr. W. T. Mckinney for making the animals and metabolism cages available 10 us. Address reprim requests to: Charles T. Snowdon, Department of Psychology, University of Wisconsin, Madison, Wisconsin 53706. injections of aldactizide (Wiener \& Deutsch, 1967; Wong \& Wilson, 1973), and adrenalectomy (Bare, 1949; Nachman, 1962; Richter, 1939; Smith, Stricker, \& Morrison, 1969). Less frequently used have been the techniques of intraperitoneal dialysis (Falk, 1961) and dietary deficiency (Nachman, 1962; Wagman, 1963). Falk (1961) has stated that a true sodium deficiency could never develop through dietary means in an animal, due to the efficiency of renal conservation of sodium. However, Wagman (1963) did find that his dietary sodium-deficient rats showed increased preferences for sodium solutions.

While there is an extensive literature dealing with both the behavioral responses toward $\mathrm{NaCl}$ solutions and the behavioral regulation of sodium balance in rats, there has been no literature on the behavioral responses to sodium in nonhuman primates, although there have been a few neurophysiological studies on the organization of macaque taste receptors (Gordon et al., 1959; Ogawa, Yamashita, Noma, \& Sato, 1972; Sato, Ogawa, \& Yamashita, 1975).

The present experiments present data on the ingestion of different concentrations of $\mathrm{NaCl}$ solutions by rhesus monkeys (Macaca mulatta) to test the "dilute water" hypothesis of Deutsch and Jones and on the response of the same animals to various $\mathrm{NaCl}$ concentrations during sodium deficiency Dietary deficiency was chosen as a means of imposing deficiency for several reasons: to avoid surgical intervention, to produce chronic rather than acute deficiency, and to provide an approximation to the conditions under which monkeys might experience sodium deficiency in their natural environments.

\section{METHOD}

The animals used were four $2 \frac{1}{2}$-year-old rhesus macaques from 
the University of Wisconsin Primate Laboratory (three females and one male), weighing $2.40-3.20 \mathrm{~kg}$ at the start of the experiment. They were housed in individual wire-mesh metabolism cages $\left(74 \mathrm{~cm}^{3}\right)$ and were maintained on a special diet consisting of $22 \%$ (by weight) sucrose, $45 \%$ puffed rice, $33 \%$ roasted soybeans, $.02 \%$ isoniazid (used prophylactically against tuberculosis), and enough deionized, distilled water to make the diet cohesive. The following vitamins were added to a 1-day ration: Abdec multiple vitamins $(.6 \mathrm{ml}$ per animal), ascorbic acid $(30 \mathrm{mg}$ per animal), and pyridoxine hydrochloride ( $2 \mathrm{mg}$ per animal). The special diet was close in nutritional and caloric value to standard monkey chow. However, the special diet contained only $2.1 \mathrm{mg}$ $\mathrm{Na}$ per $100 \mathrm{~g}$ of diet. Two hundred and twenty milligrams $\mathrm{NaCl}$ per $100 \mathrm{~g}$ were added to produce the nondeficient diet (equivalent to the $\mathrm{Na}$ concentration in standard monkey chow). Deionized distilled water was used in making the diet, the test solutions, and for general nontest drinking. The test solutions consisted of distilled water, $.45 \% \mathrm{NaCl}, .9 \% \mathrm{NaCl}, 1.35 \% \mathrm{NaCl}$, or $1.8 \% \mathrm{NaCl}$.

The animals were placed on the nondeficient diet for 7 days. Then each subject received the test solutions for 20 days, one solution per day in a randomized Latin square design (each of the five test solutions was presented four times). The test solutions were presented at 12:00 noon and removed at 5:00 p.m., when the animals were given fresh food and distilled water. The test solutions were present for only $5 \mathrm{~h}$ in order to carefully monitor intake, to maximize sodium intake over a short time period, and to allow animals to hydrate themselves between test periods if they refused to drink the salt solutions.

Following the 20 days of exposure to the test solutions, the animals were fed the low $\mathrm{Na}$ diet $(2.1 \mathrm{mg}$ per $100 \mathrm{~g}$ diet $)$ for 9 days. Then the test solutions were presented again on the same schedule while the monkeys continued ingesting the sodium-deficient diet. Water intake and test solution intake were recorded daily.

\section{Blood Analysis}

Blood samples $(2 \mathrm{cc})$ were collected once per week throughout the experiment and increased to every other day during the nine day sodium deprivation period and during the last 7 days. The blood was withdrawn from the femoral vein, and the serum was analyzed for sodium and potassium concentrations by flame photometry (Instrumentation Laboratories).

\section{Urine Analysis}

Urine samples were collected every $24 \mathrm{~h}$ during the last 5 days of the first test series, throughout the deprivation period, for the first 6 days of the second test solution series, and on the last 5 days of the second test solution series. The urine samples were also analyzed for sodium and potassium concentrations.

\section{Data Analysis}

Analyses of variance were performed on the group data for the following measures: $\mathrm{NaCl}$ intake as percent of total fluid intake, milliliters of $\mathrm{NaCl}$ intake, serum $\left[\mathrm{Na}^{+}\right]$, urinary $\left[\mathrm{Na}^{+}\right]$, and urinary $\left[\mathrm{K}^{+}\right] /\left[\mathrm{Na}^{+}\right]$. Subsequent analyses of variance were performed on each individual's data. Planned comparison t tests were used to evaluate differences between means.

\section{RESULTS}

\section{Behavioral Response to Sodium Solutions}

In the behavioral data for $\mathrm{NaCl}$ intake as a percent of total intake, there was a main effect for concentration $[F(4,12)=11.43, p<.001]$. Consumption of distilled water $(0 \% \mathrm{NaCl})$ was significantly greater than consumption of any of the salt solutions [ts(3) $>3.21$, ps $<.05$ ). The data are presented for each individual in the solid curves of Figures 1 and 2 .

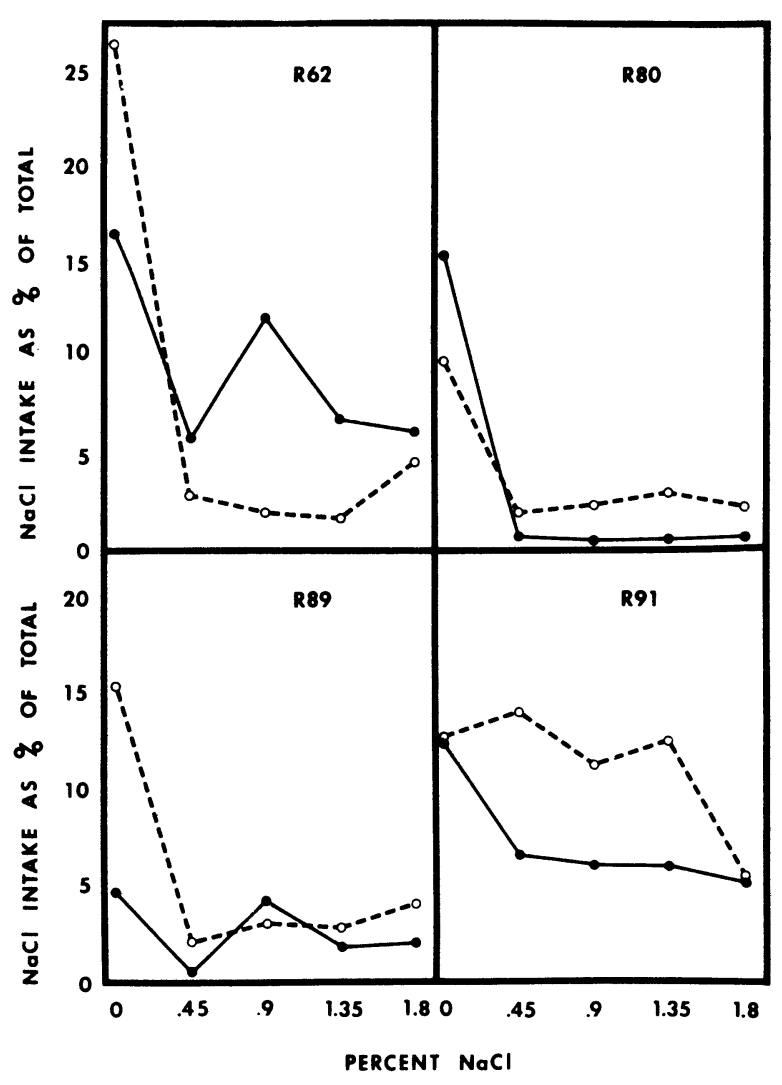

Figure 1. Test solution intake as a percent of $24-h$ total intake for each animal. Solid lines indicate the predeficiencs phase; dotted lines indicate the sodium-deficiency phase.

There was no indication of a preference-aversion curve for any of the animals, and for each animal the intake of distilled water was greater than that of any concentration of $\mathrm{NaCl}$.

\section{Behavioral and Physiological Responses to Sodium Deficiency: Group Analyses}

There was no effect of sodium deficiency on group measures of sodium intakes. There was no evidence for either increased sodium solution intake after deficiency or for a preference-aversion curve. Sodium deficiency did have an effect on physiological measures. There was a marginally significant effect on serum sodium concentration $[\mathrm{F}(2,6)=4.82$, $\mathrm{p}<.06$, with comparison of means showing a reduction in serum sodium during the sodiumdeficiency phase $[\mathrm{t}(3)=2.59, \mathrm{p}<.10]$. There was a significant effect on urinary sodium concentration $[\mathrm{F}(2,6)=9.23, \mathrm{p}<.025]$. Comparison of means showed that the predeficiency urinary values were significantly greater than both the phase of sodium deficiency $[\mathrm{t}(3)=3.34, \mathrm{p}<.05]$ and the phase of sodium deficiency with sodium chloride solutions available $[\mathrm{t}(3)=6.86, \mathrm{p}<.01]$. There were no significant effects with the analysis of urinary potassium/sodium ratios, a bioassay of aldosterone 


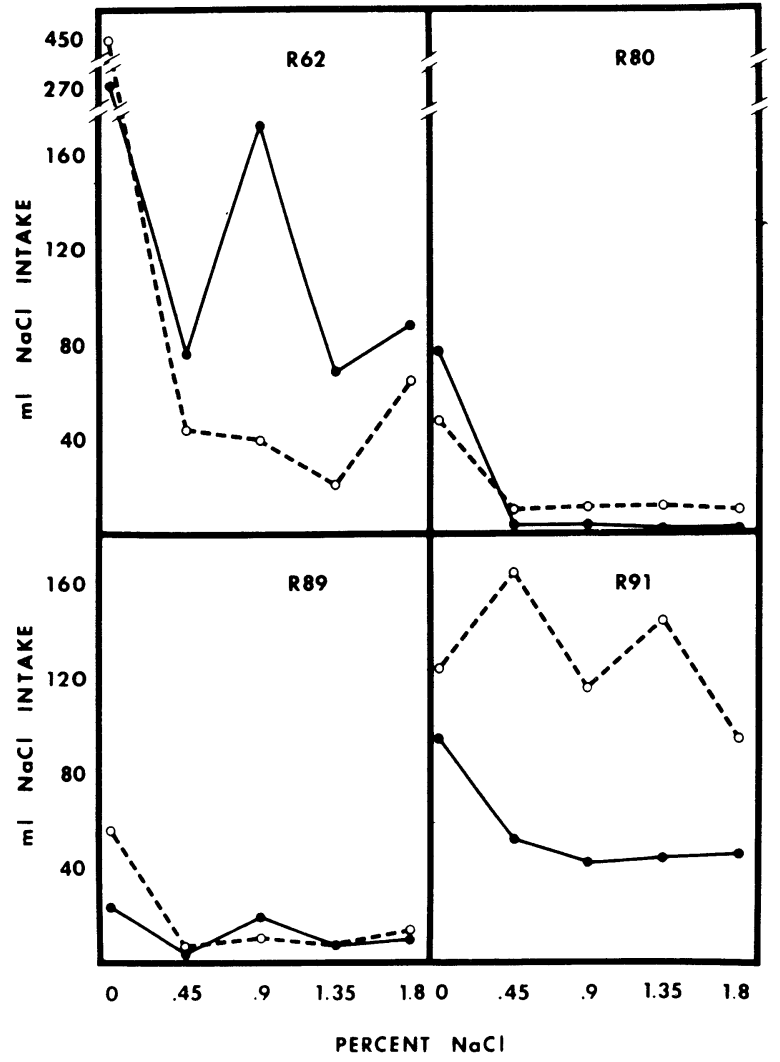

Figure 2. Test solution intake in milliliters for each animal. Solid lines indicate the predeficiency phase; dotted lines indicate the sodium-deficiency phase.

secretion (Jalowiec \& Stricker, 1970; Jalowiec, Stricker, \& Wolf, 1970).

\section{Behavioral and Physiological Responses to Sodium Deficiency: Individual Analyses}

There were no changes in grouped data in $\mathrm{NaCl}$ ingestion as a function of sodium deficiency, even though urinary and serum sodium concentrations indicated that a physiological sodium deficiency existed. Because of the failure to find expected behavioral changes resulting from sodium deficiency, we analyzed the data of individual animals using a within-subjects analysis of variance based on the four replicate behavioral measures taken in each phase of the study, and on four replicate samples for each physiological measure taken at equivalent time periods during each phase of the study. The data of the individual animals are presented in Figures 1-3. Figure 1 presents $\mathrm{NaCl}$ solution intake as a percentage of the total $24-\mathrm{h}$ fluid intake. Figure 2 presents the milliliters of $\mathrm{NaCl}$ solution ingested over the 5 -h test period. Figure 3 presents the mean serum sodium concentrations, urinary sodium concentrations, and urinary potassium/sodium concentration ratios for each animal. Three different response patterns were found among the four subjects.
R 62. This animal showed significant effects of test solution intake as percent of total intake for both treatment $[\mathrm{F}(1,3)=13.12, \mathrm{p}<.05]$ and concentration $[F(4,12)=30.07, p<.001]$. Comparison of means indicated significantly greater intakes of $.9 \%$ and $1.8 \% \mathrm{NaCl}$ solutions in the nondeficient phase than in the sodium deficiency phase [ts(3) $>3.74$, ps $<.05$ ]. When the milliliters of test solution intake were analyzed, there were also significant effects for concentration $[F(4.12)=29.02$, $\mathrm{p}<.001]$ and the Concentration by Treatment interaction $[\mathrm{F}(4,12)=5.99, \mathrm{p}<.01]$. Comparison of means for this measure also indicated increased intakes of $.9 \%$ and $1.8 \% \mathrm{NaCl}$ solutions in the nondeficient phase relative to the deficient phase $[\operatorname{ts}(3)$ $>3.13$, ps $<.06$ ]. This is the reverse of the expected finding of increased sodium ingestion following deficiency.

Analyses of physiological measures indicated that serum sodium concentrations were lower in the period of sodium deficiency alone than either the predefiency phase $[\mathrm{t}(3)=6.95, \mathrm{p}<.025]$ or the deficiency plus sodium solution phase $[\mathrm{t}(3)=5.43$, $\mathrm{p}<.025]$. Urinary sodium concentrations were marginally reduced from the predeficiency to the deficiency phase $[\mathrm{t}(3)=2.55, \mathrm{p}<.10]$, while the deficiency plus sodium solution phase did not differ significantly from either of the other periods. There

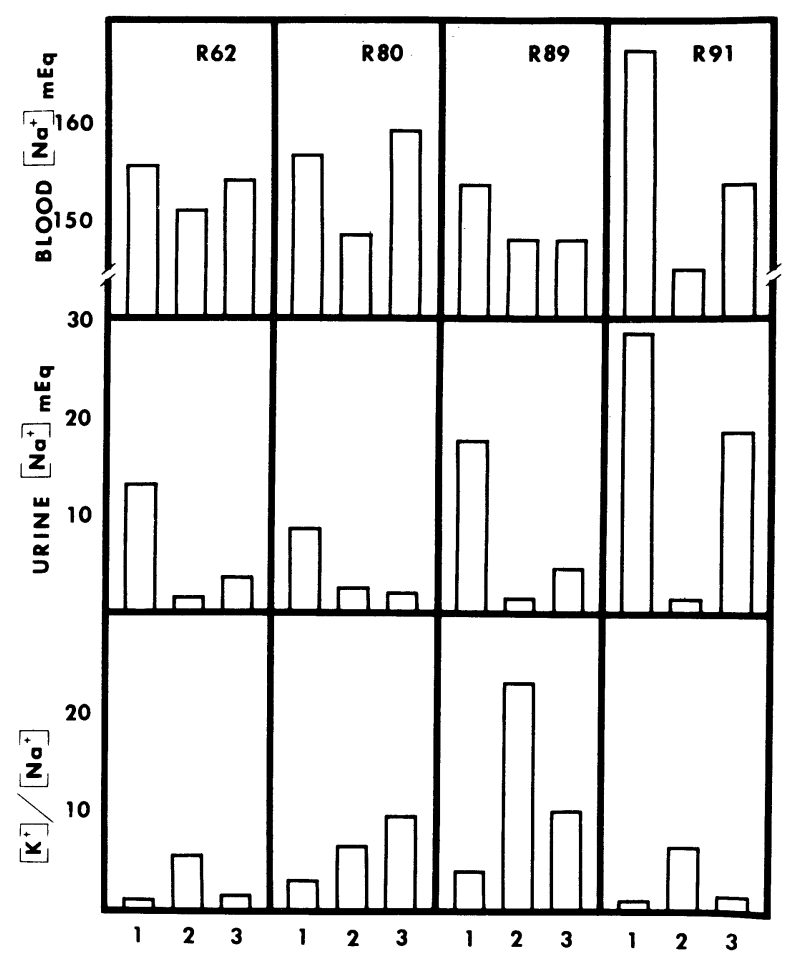

Figure 3. Serum sodium levels, urinary sodium levels, and urinary potassium-sodium ratios for each animal. $1=$ predeficiency phase: 2 = sodium deficiency without sodium ingestion phases; 3 = sodium deficiencs with sodium ingestion phase. 
was a significant effect of treatment on urinary potassium-sodium ratios $[\mathrm{F}(2,6)=14.97, \mathrm{p}<.005]$. The ratios during the deficiency stage were significantly elevated over both the predeficiency phase $[\mathrm{t}(3)=3.88, \mathrm{p}<.05]$ and the deficiency plus sodium solution phase $[\mathrm{t}(3)=3.94, \mathrm{p}<.05]$.

Thus, this animal showed a paradoxical decrease in sodium chloride ingestion following deficiency, but this decreased intake was still sufficient to restore serum sodium to predeficiency levels. Urinary sodium concentration showed a very slight increase following sodium availability, and aldosterone secretion $(\mathrm{K} / \mathrm{Na}$ ratios) returned to predeficiency levels.

R 80. The behavioral data for this animal indicated no changes as a function of sodium deficiency. The only significant effects were for concentration in both percentage intake $[F(4,12)=10.90, p<.001]$ and milliliter intake $[\mathrm{F}(4,12)=10.91, \mathrm{p}<.001]$. In both analyses, the intake of distilled water $(0 \% \mathrm{NaCl})$ was significantly greater than all other solutions.

There were significant effects for serum sodium, with the deficiency phase having lower concentration than the predeficiency phase $[\mathrm{t}(3)=3.00$, $\mathrm{p}<.10]$ and the deficiency plus sodium phase $[\mathrm{t}(3)$ $=18.45, \mathrm{p}<.001]$. This return to normal serum levels was primarily a function of control over urinary excretion of sodium. Urinary sodium was significant $[\mathrm{F}(2,6)=6.30, \mathrm{p}<.05]$, with the predeficiency levels being marginally higher than both the deficiency phase $[t(3)=2.44, p<.10]$ and the deficiency plus sodium phase $[\mathrm{t}(3)=2.71, \mathrm{p}<.10]$. Urinary potassium-sodium ratios showed a significant treatment effect $[F(2,6)=13.43, p<.01]$. Comparisons of means indicated that predeficiency ratios were lower than the deficiency plus sodium ratios $[t(3)=8.60, p<.01]$, and the deficiency ratios were marginally lower than the deficiency plus sodium ratios $[\mathrm{t}(3)=2.61, \mathrm{p}<.10]$.

This animal showed no change in sodium ingestion as a function of sodium deficiency, but did show a return to predeficiency serum sodium levels when $\mathrm{NaCl}$ solutions were available during deficiency. This seems to have been produced by the maintenance of decreased urinary sodium excretion which was in turn paralleled by continuing high levels of aldosterone secretion.

R 89. Behavioral measures on this animal indicated significant effects on percentage intake of both concentration $[\mathrm{F}(4,12)=4.83, \mathrm{p}<.025]$ and Concentration by Treatment $[\mathrm{F}(4,12)=5.23$, $\mathrm{p}<.025]$. Rather than indicating an increased ingestion of sodium following sodium deficiency, however, tests of means indicated only that the ingestion of distilled water $(0 \% \mathrm{NaCl})$ was significantly greater following sodium deficiency than before $[\mathrm{t}(3)=3.39, \mathrm{p}<.05]$. A significant concentration effect was also found for milliliters intake $[F(4,12)$ $=9.97, \mathrm{p}<.001]$. Both concentration effects were due to significantly higher levels of water ingestion and not to increased $\mathrm{NaCl}$ ingestion.

Physiological measures indicated no significant differences in serum sodium levels and a significant treatment effect in urinary sodium levels $[F(2,6)$ $=18.13, \mathrm{p}<.01]$. Means tests indicated that predeficiency levels were significantly higher than both deficiency levels $[\mathrm{t}(3)=4.57, \mathrm{p}<.02]$ and deficiencyplus-sodium levels $[\mathrm{t}(3)=3.39, \mathrm{p}<.05]$. There was a significant treatment effect of potassium-sodium ratios $[\mathrm{F}(2,6)=6.05, \mathrm{p}<.05]$, with predeficiency ratios being lower than both deficiency ratios $[\mathrm{t}(3)$ $=3.27, \mathrm{p}<.05]$ and deficiency-plus-sodium levels $[\mathrm{t}(3)=2.86, \mathrm{p}<.10]$.

This animal showed results very similar to those of $R$ 80. Neither ingested very much sodium chloride; neither increased their intakes in response to sodium deficiency; both appear to have regulated sodium balance through decreased urinary excretion and increased aldosterone secretion. The main difference between them was the failure for any changes to appear in serum sodium levels in $R 89$, while $R 80$ showed a decrease in serum sodium levels following sodium deficiency and a subsequent return to normal levels when sodium solutions were made available.

$R$ 91. The behavioral measures on this animal produced a significant Treatment by Concentration interaction on the milliliter intake measure $[F(4,12)$ $=3.39, \mathrm{p}<.05]$. Following sodium deficiency, increased intakes appeared at $.9 \% \mathrm{NaCl}[\mathrm{t}(3)=4.01$, $\mathrm{p}<.05$ ], with a marginally significant increase at $1.35 \% \mathrm{NaCl}[\mathrm{t}(3)=2.90, \mathrm{p}<.10]$. (Although Figure 2 shows elevated intakes at all concentrations following deficiency, the variability was too high to produce significance at all concentrations.) Thus, this animal showed part of the predicted behavioral response of increased sodium ingestion following sodium deficiency.

Physiological measures showed a significant treatment effect for serum sodium $[F(2,6)=16.51$, $\mathrm{p}<.025]$, with the deficiency phase levels being significantly lower than both the predeficiency phase $[\mathrm{t}(3)=5.02, \mathrm{p}<.02]$ and the deficiency-plussodium phase $[\mathrm{t}(3)=8.70, \mathrm{p}<.01]$. There was a marginally significant treatment effect for urine sodium $[F(2,6)=4.60, p<.06]$, with deficiency levels being lower than both predeficiency $[\mathrm{t}(3)=$ $3.57, \mathrm{p}<.05]$ and deficiency plus sodium $[\mathrm{t}(3)=$ $2.70, \mathrm{p}<.10$ ]. Predeficiency levels were also significantly greater than deficiency-plus-sodium levels $[\mathrm{t}(3)=3.39, \mathrm{p}<.05]$, although this was the only animal to show such high levels of urinary sodium excretion during the deficiency-plus-sodium phase. Urinary potassium-sodium ratios were also signifi- 
cant $[\mathrm{F}(2,6)=15.20, \mathrm{p}<.005]$. The deficiency ratios were significantly higher than both the predeficiency ratios $[\mathrm{t}(3)=4.05, \mathrm{p}<.05]$ and the deficiency-plus-sodium ratios [t $(3)=3.81, \mathrm{p}<.05]$.

This animal was the only one to show an increased ingestion of sodium chloride solutions following sodium deficiency. It showed a restoration of serum sodium levels and high levels of urinary sodium secretion during this deficiency-plus-sodium phase. Aldosterone levels, in contrast to animals $R 80$ and $\mathrm{R} 89$, returned to normal levels during the deficiencyplus-sodium phase.

\section{DISCUSSION}

In contrast to the results typically reported for rats of a preference-aversion curve for sodium chloride intake with a peak at isotonic saline, rhesus monkeys did not show a preference-aversion curve for $\mathrm{NaCl}$. This failure to find a preference-aversion curve for $\mathrm{NaCl}$ in rhesus monkeys is exactly what one would predict from the Deutsch and Jones (1960) hypothesis. The rhesus monkey with separate water and salt sensory pathways is not subjected to the conflicting information that the rat receives when drinking saline solutions. Distilled water was always ingested in greater volumes than saline solutions by monkeys, exactly what one would expect a thirsty animal to do.

Also in contrast to results typically reported for rats of an increased ingestion of $\mathrm{NaCl}$ solution following sodium deficiency, rhesus monkeys did not show a consistent increased $\mathrm{NaCl}$ intake following sodium deficiency. Measures of serum sodium indicated that the animals were experiencing some effects of a sodium deficiency. The failure to find differences in sodium intakes as a function of sodium deficiency in the grouped data became less puzzling with the examination of individual animals. Three separate response patterns to sodium deficiency were observed. One animal ( $R$ 91) displayed the predicted behavior of increased sodium ingestion following sodium deficiency. With the increased sodium ingestion came a return to normal serum sodium levels and a nearly normal return of urinary sodium levels accompanied by a reduction in aldosterone secretion. Two other animals ( $R$ 80, R 89) showed no behavioral response to sodium deficiency and, indeed, gave very little evidence of much sodium ingestion at all. However, they defended serum sodium levels by reducing urinary sodium levels and maintaining low levels of sodium excretion even when sodium solutions were available. They displayed increased levels of aldosterone secretion which were maintained during the time that sodium solutions were available for ingestion. The final animal ( $R$ 62) showed a reduced intake of sodium solutions following sodium deficiency and, while serum sodium levels were defended and urinary excretion of sodium remained low during the period of sodium availability, aldosterone secretion was also reduced to a low level. Despite the significant reduction in sodium intake, the animal was still ingesting greater volumes of $\mathrm{NaCl}$ than either $\mathrm{R} 80$ or $\mathrm{R} 89$. Thus, it might have had a sufficient sodium intake to allow regulation to occur despite a reduction in aldosterone secretion. The very low $\mathrm{NaCl}$ intakes of R 80 and $R 89$ probably necessitated the continued high levels of aldosterone secretion to maintain sodium balance.

The results from the grouped data could be taken as supporting Falk's (1961) contention that "it is difficult to deplete the adult animal of sodium by feeding a deficient diet. With a diet lacking in sodium, the renal excretion of sodium rapidly falls to such a miniscule value that a true depletion may never develop." (p. 201). That renal conservation is a powerful mechanism for responding to sodium deficiency is illustrated by the two animals ( $R 80$ and $R$ 89) which ingested very small amounts of sodium solution. The average daily intake of sodium of these two animals was $35.7 \mathrm{mg}$. The mean total fluid ingested daily by these animals was approximately $330 \mathrm{ml}$. If one assumes that all of this fluid was excreted as urine, then, with an average sodium excretion rate of $3 \mathrm{mEq} /$ liter (Figure 3), the total sodium excretion was $23 \mathrm{mg}$ of sodium per day. Thus, despite the very low amount of sodium ingested each day, renal conservation of sodium produced a net daily gain of $13 \mathrm{mg}$ of sodium (some of which was undoubtedly lost through perspiration). There are no minimal standards for sodium intake in rhesus monkeys (National Academy of Science, 1972), but it would seem, from the present results, that $36 \mathrm{mg}$ of sodium per day is adequate for adolescent monkeys. In the natural environment, neither rats nor rhesus monkeys are likely to experience adrenal insufficiency, peritoneal dialysis, or subcutaneous Formalin injections as often as they are likely to suffer from a dietary sodium deficiency. Thus, it is of importance to examine the types of responses, both physiological and behavioral, that organisms make to dietary sodium deficiency.

The finding of three separate adaptations to sodium deficiency is of considerable interest. In the physiologically intact organism there are two major responses to sodium defiency: conserving urinary sodium loss through increased aldosterone secretion and/or ingesting increased amounts of sodium. These strategies account for three of our four monkeys. The fourth (R 62) adopted an intermediate response of ingesting just enough $\mathrm{NaCl}$ to not require an increased aldosterone secretion to further inhibit urinary excretion. Thus the appearance of separate responses in different animals ought not to be surprising. However, the small sample sizes that 
are available for primate research do make the occurrence of individual differences more obvious than would be the case with the large sample sizes used in rodent research.

It is possible that a longer sodium deprivation period or a more severe depletion procedure before sodium testing would have produced more evidence of behavioral responses to sodium solutions. And it is possible, though not likely, that some other testing procedure, such as a two-bottle test or longer exposures to sodium solutions, might have provided more evidence of a taste aversion curve. However, the present data do indicate that monkeys respond to sodium solutions differently than rats and that, in primates, the examination of individual differences is quite important.

\section{REFERENCES}

BARE, J. K. The specific hunger for sodium chloride in normal and adrenalectomized white rats. Journal of Comparative and Physiological Psychology, 1949, 42, 242-253.

DEUTSCH, J. A., \& Jones, A. D. Diluted water: An explanation of the rat's preference for saline. Journal of Comparative and Physiological Psychology, 1960, 53, 122-127.

FALK, J. L. The behavioral regulation of water-electrolyte balance. In Nebraska Symposium on Motivation (Vol. 9). Lincoln: University of Nebraska Press, 1961. Pp. 1-33.

Gordon, G., Kitchell, R., Ström, L., \& Zotterman, Y. The response pattern of taste fibers in the chorda tympani of the monkey. Acta Physiologica Scandinavica, 1959, 46, 119-132.

JALOWIEC, J. E., \& STricker, E. M. Restoration of body fluid balance following acute sodium deficiency in rats. Journal of Comparative and Physiological Psychology, 1970, 70, 94-102.

Jalowiec, J. E., STricker, E. M., \& Wolf, G. Restoration of so- dium balance in hypophysectomized rats after acute sodium deficiency. Physiology and Behavior, 1970, 5, 1145-1149.

Moox, D. G. Oral and postingestional determinants of the intake of various solutions in rats with esophageal fistulas. Journal of Comparative and Physiological Psychology, 1963, 56, 645-659.

NaChMAN, M. Taste preferences for sodium salts by adrenalectomized rats. Journal of Comparative and Physiological Psychology, 1962, 55, 1124-1129.

National Academy of Science, Subcommitee on LaboraTORY ANIMAL NUTRITION. Nutritional requirements of laboratory animals. Washington, D.C., 1972.

Ogawa, H., Yamashita, S., Noma, A., \& Sato, M. Taste responses in the macaque monkey chords tympani. Physiology and Behavior, 1972, 9, 325-331.

Richter, C. P. Salt taste thresholds of normal and adrenalectomized rats. Endocrinology, 1939, 24, 367-371.

Sato, M., Ogawa, H., \& Yamashita, S. Response properties of macaque monkey chorda tympani fibers. Journal of General Physiology, 1975, 66, 781-810.

SMith, D. F., STricker, E. M., \& Morrison, G. R. NaCl solution acceptability by sodium deficient rats. Physiology and Behavior, 1969, 2, 239-243.

STricker, E. M. Extracellular fluid volume and thirst. American Journal of Physiology, 1966, 211, 232-238.

W AGMAN, W. Sodium chloride deprivation: Development of sodium chloride as a reinforcement. Science, 1963, 140, 1403-1404.

Wiener. N., \& Deutsch, J. A. Effects of salt deprivation and strain differences on tests of the diluted water hypothesis. Journal of Comparative and Physiological Psychology, 1967, 64, 400-403.

Wong, R., \& Wilson, C. S. Aldactazide-induced sodium appetite in rats. Behavioral Biology, 1973, 8, 285-289.

Zotterman, Y. Species differences in the water taste. Acta Physiologica Scandinavica, 1956, 37, 60-70.

(Received for publication August 4, 1977; revision accepted October $4,1977$. 